\title{
Ocean sound channel ray path perturbations from internal-wave shear and strain
}

\author{
Timothy F. Duda \\ Applied Ocean Physics and Engineering Dept., MS 11, Woods Hole Oceanographic Institution, \\ Woods Hole, Massachusetts 02543
}

(Received 16 December 2004; revised 13 July 2005; accepted 1 August 2005)

\begin{abstract}
The relative importance of internal-wave strain and internal-wave shear on perturbation of acoustic ray trajectories in the ocean is analyzed. Previous estimates based on the Garrett-Munk internal-wave spectral model are updated using data from recent field studies of internal waves. Estimates of the ratio of the rms shear effect to the rms strain effect based on data from the upper kilometer of ocean are as high as $0.25-0.4$, exceeding the estimates of $0.08-0.17$ stemming from the model. Increased strength of three phenomena that have shear to strain ratios higher than the internal-wave average can cause this effect. These are near-inertial waves, internal tides, and vortical modes. (C) 2005 Acoustical Society of America. [DOI: 10.1121/1.2062127]
\end{abstract}

PACS number(s): 43.30.Cq, 43.30.Ft, 43.30.Re [WLS]

Pages: 2899-2903

\section{INTRODUCTION}

Small-scale temporally variable perturbations to the average ocean sound channel have been known for some time to cause acoustic field variability. Based on work motivated by reciprocal-transmission measurement of currents [Worcester (1977)], studies of variability generally neglect the effect of fluctuating current shear with respect to the effect of the fluctuating vertical derivative of sound-speed [Colosi, Flatté, and Bracher (1994); Flatté (1983) Sec. II D; Munk, Worcester, and Wunsch (1995)]. Those studies use the Garrett-Munk (GM) spectral model of internal waves to describe perturbation strain in the ocean. [GM describes both strain and shear in the case of Worcester (1977)]. The strain is responsible for sound-speed perturbations. However, measurements from the ocean suggest that conditions may vary from GM. In particular, stronger shear to strain ratios have been observed than are consistent with GM, so the relative strength of the two terms is revisited here and in another paper [Colosi (2005)].

The relative effects of strain and shear on ray-path perturbations are chosen for analysis, rather than some other aspect of the acoustic field, because a ramification of ocean medium fluctuations is temporally variable ray-path alteration, which is one manner of describing acoustic field fluctuations [Simmen, Flatté, and Wang (1997)]. Also, the path perturbations themselves have been suggested to be an important indicator of sound field variability (Beron-Vera and Brown (2003); Brown et al. (2003); Brown and Viechnicki (1998); Smith, Brown, and Tappert (1992); Virovlyansky (2003))]. Ray paths can fluctuate rapidly because of two internal wave effects: changes to the vertical gradient of sound-speed caused by internal-wave vertical strain, and to the vertical component of internal-wave shear.

Here, it is reported that the rms shear effect on rays may be as large or larger than one-quarter the rms strain effect, which is roughly double the $13 \%$ effect predicted using GM. Both ratios refer to effects on rays in the upper ocean, where both effects will have their maximum effect on rays. This means that it may be important to include shear effects in some situations. For completeness, expressions that govern ray trajectories in the presence of internal wave currents that have an angle/depth form are derived. This form facilitates comparison by having terms related to shear and strain in only one equation. Next, the rms magnitudes of various perturbation terms controlling ray curvature are examined as functions of depth in the ocean sound channel, and are compared with curvature imposed by the mean profile of sound speed.

The paper is organized in the following way. In Sec. II, the ray equations valid in a moving medium in the form appropriate for the deep-ocean sound channel are given. In Sec. III, the sizes of various perturbation terms in the ray angle equation are investigated, showing the relative importances of various small-scale effects. Results obtained using the GM model and using oceanic data are computed and are compared to each other. Sec. IV is a summary.

\section{RAY TRAJECTORIES IN A MOVING INHOMOGENEOUS MEDIUM}

The analysis uses an angle/depth version of the equations governing ray trajectories in an inhomogeneous medium with inhomogeneous motion. These equations can be derived using the calculus of variations and Fermat's principle of least time. This approach is outlined in one reference [Munk, Worcester, and Wunsch (1995)], and appears in a report [Bowlin et al. (1993)] (The report may be found online). To apply Fermat's principle to long-range propagation in the ocean sound channel, we must assume that the ray approximation, as derived from Helmholtz equation via the eikonal equation [Ostashev (1997), for example], a highwavenumber model, provides a valid description of the physics at the frequency of interest. The ray approximation has been shown to hold at frequencies as low as $75 \mathrm{~Hz}$ [Colosi et al. (1999)].

Mathematically, Fermat's principle is expressed by setting the variation of the time to zero, 


$$
\delta T=\delta \int d t=0,
$$

where the integral on the right hand side is over the path of the ray with fixed limits of integration and where $\delta$ is any (differentiable) variation in the path that keeps the end points fixed. In order to find the path we convert the integral over $d t$ to an integral over path length $d s$ using the slowness in the moving medium

$$
Q=d t / d s=1 /\left(c+u_{s}\right)=1 /\left(c+u_{r} \cos \theta+u_{z} \sin \theta\right),
$$

where $c$ is the sound speed, $u_{r}$ and $u_{z}$ are medium velocity components along horizontal $r$ axis and vertical $z$ axes, and $u_{s}$ is the medium velocity in the direction of the ray. $\theta$ is the angle between the ray and the horizontal. Out-of-plane velocity is disregarded. This gives

$$
\delta T=\delta \int Q d s .
$$

The path length can be expressed in terms of Cartesian coordinates $x_{i}$ as $d s=\left(d x_{i} d x_{i}\right)^{1 / 2}$ where we use the summation convention of implicitly summing over repeated indices.

Using manipulations common in the calculus of variations yields

$$
\delta T=\int \delta x_{i}\left(\partial_{i} Q-\frac{d}{d s}\left(Q \dot{x}_{i}\right)\right) d s,
$$

where a dot over a quantity indicates the total derivative of the quantity with respect to $s$. For this expression to be zero for any and all variations, the term within parentheses must equal zero. This yields the ray equation, which may be also be written

$$
\ddot{x}_{i}=\frac{\partial_{i} Q}{Q}-\dot{x}_{i}\left(\dot{x}_{j} \frac{\partial_{j} Q}{Q}\right) .
$$

The components $\dot{x}_{i}$ form a unit vector pointing along the direction of the ray. The second term on the right hand side is the projection of $\nabla Q / Q$ in the $\dot{x}$ direction. Thus $\ddot{x}$ is equal to that part of $\nabla Q / Q$ which is perpendicular to the path of the ray.

To put this into a more familiar form used in ocean acoustics, and applying the typically used restriction of propagation only within a plane, consider the ray angle $\theta$. The $r$ component of the resulting ray equation is an expression for $\dot{\theta}$,

$$
Q \dot{\theta}=\partial_{z} Q \cos \theta-\partial_{r} Q \sin \theta .
$$

The slowness $S=1 / c$ is typically used in derivations of this type, instead of the quantity $Q$ used here. The inclusion of medium velocity in the slowness implies that an advective push of the sound does not invalidate Fermat's principle, which may be intuitive. Intuition does not constitute a proof, however. Fortunately, a proof that Fermat's principle is valid for a moving medium has recently been published [Godin and Voronovich (2004)].

A few manipulations yield

$$
\begin{aligned}
\frac{d \theta}{d r}= & -Q\left(\partial_{z} c+\partial_{z} u_{r} \cos \theta+\partial_{z} u_{z} \sin \theta\right) \\
& +Q \tan \theta\left(\partial_{r} c+\partial_{r} u_{r} \cos \theta+\partial_{r} u_{z} \sin \theta\right) .
\end{aligned}
$$

Now, expand $Q$ with $u_{i} / c$ as a small parameter, giving

$$
\begin{aligned}
\frac{d \theta}{d r}= & -c^{-1}\left(1-u_{r} \cos \theta / c-u_{z} \sin \theta / c\right)\left(\partial_{z} c+\partial_{z} u_{r} \cos \theta\right. \\
& \left.+\partial_{z} u_{z} \sin \theta\right)+c^{-1} \tan \theta\left(1-u_{r} \cos \theta / c-u_{z} \sin \theta / c\right) \\
& \times\left(\partial_{r} c+\partial_{r} u_{r} \cos \theta+\partial_{z} u_{z} \sin \theta\right) .
\end{aligned}
$$

Eighteen terms remain on the right hand side. With no motion this is the basic ray angle equation. The other terms show the effects of advection, which are small in the ocean. If the sound speed is written as a basic profile plus perturbations $c(r, z)=c_{o}(z)+c^{\prime}(r, z)$, and if we delete most of the derivatives with respect to range, which are smaller than derivatives with respect to vertical because the ocean is known to have anisotropic perturbations, then we can write an expression that includes the effects of velocity and sound speed perturbations

$$
\begin{aligned}
\frac{d \theta}{d r}= & -c_{o}^{-1}\left(1-c^{\prime} / c_{o}-u_{r} \cos \theta / c_{o}-u_{z} \sin \theta / c\right)\left(\partial_{z} c_{o}\right. \\
& \left.+\partial_{z} c^{\prime}+\partial_{z} u_{r} \cos \theta+\partial_{z} u_{z} \sin \theta-\partial_{r} c_{o} \tan \theta\right),
\end{aligned}
$$

where terms of higher order in $c^{\prime} / c_{o}$ have been omitted. Further analysis in the next section shows the relative influences of the anomaly $c^{\prime}$, the shear $\partial_{z} u_{r}$, the strain $\partial_{z} u_{z}$, and the velocities $u_{r}$ and $u_{z}$.

An additional equation needed to trace rays is that for ray height

$$
\frac{d z}{d r}=\tan \theta .
$$

If travel time is of interest, it is given to first order in $u_{i} / c$ by this expression:

$$
\frac{d t}{d r}=\frac{\sec \theta}{c}\left(1-u_{r} \cos \theta / c-u_{z} \sin \theta / c\right) .
$$

The advective effect of the medium velocity enters in an intuitive way.

Note that (9)-(11) have the same meaning as the sets of equations appearing in other works [Franchi and Jacobson (1972); Lamancusa and Daroux (1993); Ostashev (1997)], which might have been chosen for analysis instead. However, the form used here contains medium properties in only one of the two geometry equations, facilitating the analysis.

\section{COMPARISON OF PERTURBATION TERMS}

To first order in $u_{i} / c_{o}$ and $c^{\prime} / c_{o}$, rewrite (9) as

$$
\begin{aligned}
\frac{d \theta}{d r}= & -c_{o}^{-1}\left[\partial_{z} c_{o}\left(1-c^{\prime} / c_{o}-u_{r} \cos \theta / c_{o}-u_{z} \sin \theta / c_{o}\right)\right. \\
& \left.+\partial_{z} c^{\prime}+\partial_{z} u_{r} \cos \theta+\partial_{z} u_{z} \sin \theta-\partial_{r} c_{o} \tan \theta\right] .
\end{aligned}
$$

Here, we have taken advantage of the fact that small-scale ocean sound-speed and velocity perturbations have red spec- 
tra [Garrett and Munk (1975); Pinkel (1984)], so that most of the energy is at low wavenumbers, below order $2 \pi /(500 \mathrm{~m})$ in the horizontal and order $2 \pi /(100 \mathrm{~m})$ in the vertical. This means that the differentiation operation on perturbations (multiplication by wavenumber for members of a Fourier expansion) reduces magnitude, so that many derivative terms have been omitted.

There are eight terms on the right-hand side of (12). Although many small terms have already been neglected, many remaining terms are typically also quite small and could sensibly be omitted for almost all ocean acoustic situations. There is no danger in retaining these terms, however, although their presence may obscure the essential physics. The danger lies in incorrectly omitting significant terms. The first and last terms in (12) represent the effect of the unperturbed sound channel. Between them are six perturbation terms. The first three perturbation terms are corrections to the rate of change of $\theta$ versus $r$ that arise because the sound is not traveling at the background speed $c_{o}$. These are very small and are not considered any further. Term 7 is expected to be much less than term 2 and is also not evaluated. (The ratio of term 7 to term 2 for a wave of angular frequency $\omega$ is $\omega / \partial_{z} c_{0}$.) The relative sizes of the remaining perturbation terms $\left(5\right.$ and $6, \partial_{z} c^{\prime}$ and $\left.\partial_{z} u_{r} \cos \theta\right)$ are examined in the next section using two methods: Computations involving GM model internal-wave spectra, and evaluation in terms of directly observed small-scale velocities and sound-speed perturbations. Because a number of papers have shown that realistically scaled perturbations $c^{\prime}$ can lead to large ray path alterations about a mean state [Beron-Vera and Brown (2003); Virovlyansky (2003)], we must only show that $\partial_{z} u_{r} \cos \theta$ is similar to $\partial_{z} c^{\prime}$ in magnitude in order to verify its importance.

\section{A. Analysis of terms using a spectral model}

The GM model spectrum, which fits observed internal wave spectra in most regions of the deep ocean (away from the uppermost $500 \mathrm{~m}$ ) within about a factor of three, provides expressions for variances of internal wave horizontal velocity $\hat{u}=\hat{r} u_{r}+\hat{y} u_{y}$, shear $d \hat{u} / d z$, displacement $\eta$, and strain $\partial_{z} \eta$. Hat indicates vector. $\hat{r}$ and $\hat{y}$ are unit vectors. The square roots of these provide useful estimates of typical perturbation term magnitudes. Worcester (1977) has already analyzed the relative sizes of terms 5 and 6 using the GM model spectrum and a canonical sound-speed profile. Only a few numbers are given, however, and subsequent work has formalized the relationship between shear variance and strain variance in the model, so a similar analysis is included here. Also, the GM model was new at the time of that publication, and details concerning how it compares with oceanic data have since been uncovered. These are discussed here.

The GM model expresses wave spectral density in terms of frequency and vertical mode number. Manipulations allow spectra in terms of frequency, horizontal wavenumber, and vertical wavenumber to also be written. The dispersion relation, which relates wavenumber angle from the horizontal to frequency, constrains the model, so that only two of those three are independent. The primary features of GM are sym-
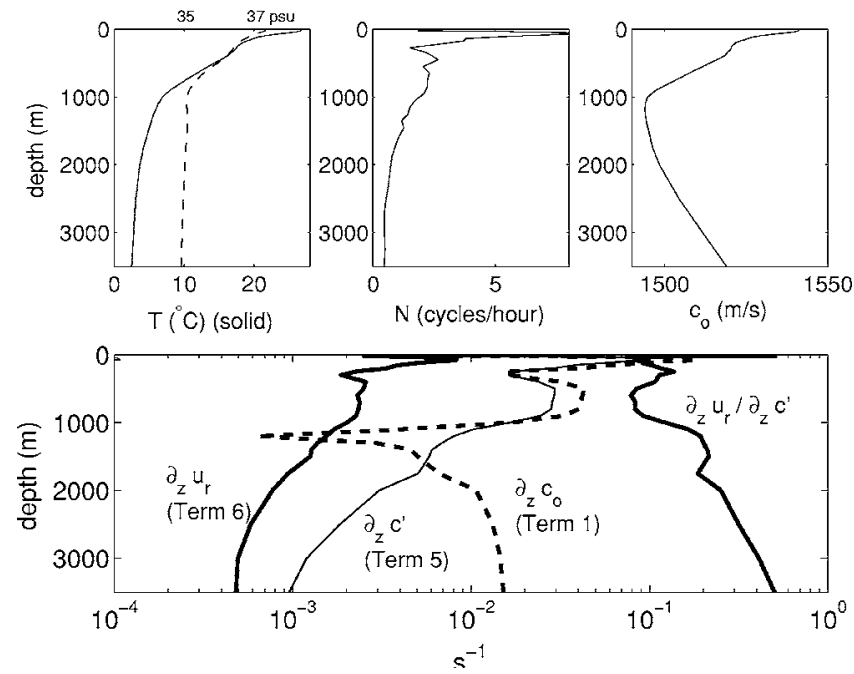

FIG. 1. The top left panel shows temperature $T(z)$ and salinity $S(z)$ (dashed line) profiles used in the GM model computation of fluctuation term rms values. The top center and top right panels show buoyancy-frequency and sound-speed profiles computed from $T(z)$ and $S(z)$. The lower panel shows rms magnitudes of terms 1 (mean profile), 5 (strain), and 6 (shear) in Eq. (12). The lower panel also shows the dimensionless ratio of shear to strain rms magnitudes at the right, as a function of depth. The shear to strain ratio $R_{\omega}$ is 3 in the GM model. Higher $R_{\omega}$ have been observed in the ocean, implying a higher ratio of shear to strain effects.

metry in vertical wavenumber, horizontal isotropy, and separable wavenumber and frequency dependencies. The fair agreement with observation stems in part from the fact that the model parameters were deduced from observations [Garrett and Munk (1975)]. Some of the differences between GM and actual ocean spectra are pertinent to this work and are discussed later.

For given temperature and salinity profiles, the GM model yields expressions for the four variances of interest that are functions of depth, with depth-dependence parameterized in terms of the buoyancy frequency $N(z)$, which is proportional to the density gradient. For this computation, profiles from the summer Levitus database for the position $27^{\circ} \mathrm{N}, 48^{\circ} \mathrm{W}$ in the North Atlantic are chosen [Levitus (1982)]. From these, $N(z)$ and $c_{o}(z)$ are computed (Fig. 1). For the GM76 version of the model, the variances are given as follows [Gregg (1989)]

$$
\begin{aligned}
& \left\langle u^{2}\right\rangle=\left\langle u_{r}^{2}\right\rangle+\left\langle u_{y}^{2}\right\rangle=(3 / 2) b^{2} E N_{0}^{2}\left(N / N_{0}\right), \\
& \left\langle\left(\partial_{z} u\right)^{2}\right\rangle_{k_{u}}=(3 / 2) \pi E b j_{*} N_{0}^{2} k_{u}\left(N / N_{0}\right)^{2}, \\
& \left\langle\eta^{2}\right\rangle=(1 / 2) E b^{2}\left(N / N_{0}\right)^{-1}, \\
& \left\langle\left(\partial_{z} \eta\right)^{2}\right\rangle_{k_{u}}=\left\langle\left(\partial_{z} u\right)^{2}\right\rangle_{k_{u}} /\left(R_{\omega} N^{2}\right),
\end{aligned}
$$

where $E=6.3 \times 10^{-5}$ is the dimensionless energy parameter, $N_{0}=5.2 \times 10^{-3}(3 \mathrm{cph}), f$ is the Coriolis frequency, $b=1300 \mathrm{~m}$ is the stratification length scale, and $j_{*}=3$ is the internal wave bandwidth parameter. The shear to strain ratio $R_{\omega}$ is 3 for the GM model. The notation \langle\rangle$_{k_{u}}$ means integrated up to cut-off vertical wavenumber $k_{u}$. The final expression stems from a different paper than the others [Polzin, Toole, and Schmitt (1995)]. Note that $b$ 
$=1300 \mathrm{~m}$ is almost universally chosen when the GM spectrum is evaluated, despite the fact that the $N(z)$ profile rarely has the form $\exp (z / 1300)$, particularly in the upper $500 \mathrm{~m}$.

Rms values of $u_{r}, \partial_{z} u_{r}, \eta$, and strain $\partial_{z} \eta$ are found by taking the square roots. Rms magnitudes of the horizontal velocity and shear terms (third and sixth) of (12) can then be computed directly. The $c^{\prime}$ terms (second and fifth) require conversion of displacement to sound speed perturbation via $c^{\prime}=\eta\left(\partial_{z} c_{o}-\gamma_{a} c_{o}\right)$ where $\gamma_{a}$ is the adiabatic sound speed gradient, typically $1.1 \times 10^{-5} \mathrm{~m}^{-1}$, and via $\partial_{z} c^{\prime} \approx \partial_{z} \eta\left(\partial_{z} c_{o}\right.$ $\left.-\gamma_{a} c_{o}\right)$

The lower part of Fig. 1 shows the magnitudes of the rms values of three of the five terms chosen for analysis, as functions of depth. Terms 2 and 3 lie off the left edge of the plot, varying between $10^{-7}$ at great depths and $10^{-4}$ at shallow depths. The shear term (number 6) is a little smaller than the $\partial_{z} c^{\prime}$ strain term (number 5). Both exceed the magnitude of the background term (number 1) near the sound channel axis. The strain term is seen to give an appreciable effect in the upper water column, consistent with the results of the papers showing ray path fluctuation cited previously. The shear term approaches the strain term in magnitude at depth, but each are smaller than $\partial_{z} c_{o}$ (term 1) there.

Shear term rms values are smaller than strain term rms values at all depths for this model, with the ratio ranging from 0.08 to 0.15 in the upper kilometer. (These are different values than obtained by Worcester using the canonical profile). However, we have two reasons to believe that the GM model underestimates shear with respect to strain. One reason is that GM does not include near-inertial waves, which have high shear and low strain, to the degree sometimes observed in the field, and does not include the spectral peaks at tidal bands (internal tides) that are apparent in some data [Nash et al. (2004)]. An excess of these types of waves at the low-frequency end of the internal-wave band would increase the shear to strain spectral ratio above the GM value of $R_{\omega}$ $=3$. (Some data sets do not provide enough information to compute $R_{\omega}=3$.) Another reason is that $R_{\omega}>3$ have been observed in the upper kilometer of water at many locations [Polzin et al. (2003)], implying that excess low-frequency waves and/or finescale velocity features such as zerofrequency geostrophically balanced vortical modes (not included in GM), may be prevalent. The shear variances at those locations exceed that of GM, while the strain variances are in line with GM. This would boost the shear term contribution to trajectory perturbations a factor of about three in the extreme cases, increasing the shear-effect to strain-effect ratios to the range 0.24 to 0.45 .

\section{B. Observational evidence}

The GM model is a useful benchmark for intercomparison of observed internal wave spectra. However, even with adjustments such as made at the end of the previous section, it does not fully describe many observed spectra, particularly those in the upper ocean [Duda and Cox (1989); Pinkel (1984); Pinkel (1985)]. The separability criterion is notably troublesome. Thus, it is prudent to estimate perturbation term sizes by looking directly at shear and strain data. Multiplication of the GM spectrum by a scaling factor so that it matches observed rms shear, for example, might not produce rms strain that also matches the observations.

Data sets providing concurrent estimates of rms shear $\partial_{z} u_{r}$ and rms $\partial_{z} c^{\prime}$ are available. One such data set was collected in the North Atlantic Tracer Release Experiment (NATRE) in the North Atlantic Ocean at $25-27^{\circ} \mathrm{N}, 28-35^{\circ} \mathrm{W}$ [Duda and Jacobs (1995); Ledwell, Watson, and Law (1998)]. Using data from a profiling float, rms shear values from a depth range of approximately $250-425 \mathrm{~m}$ were measured to be $0.005 \mathrm{~s}^{-1}$ in the fall of 1992 and $0.007 \mathrm{~s}^{-1}$ in the spring of 1993 [Duda and Jacobs (1995)]. These must be multiplied by 0.707 to get standard deviation for a single component. The resulting $\mathrm{rms} \partial_{z} u_{r}$ values of 0.0035 and $0.005 \mathrm{~s}^{-1}$ are a bit higher than the term 6 predictions of order $0.002 \mathrm{~s}^{-1}$ shown in the lower part of Fig. 1. Rms highfrequency strain values obtained from the same float were always near $\left(\partial_{z} \eta\right)_{\mathrm{rms}}=0.3 \mathrm{~s}^{-1}$. These strain values differ from the values of Polzin et al. (2003) mentioned at the end of the previous section because these do not include static finestructure signatures (possibly vortical modes), which those do (i.e., these data contain only strain and shear effects in the internal-wave frequency band). Because measured $\left|\partial_{z} c_{o}\right|$ is $\sim 0.04 \mathrm{~s}^{-1}$ and $\left|\partial_{z} c_{o}-\gamma c_{o}\right|$ is $\sim 0.06 \mathrm{~s}^{-1}$, resulting $\mathrm{rms} \partial_{z} c^{\prime}$ values are about $0.018 \mathrm{~s}^{-1}$, close to the term 5 prediction shown in Fig. 1, but only about four times larger than the higher of the two rms $\partial_{z} u_{r}$ values. Thus, the ratio of shear and strain term rms values is 0.25 rather than the GM value near 0.1 .

Consistent with this, shear to strain ratios $R_{\omega}$ measured in NATRE ranged from 7 to 19 , always exceeding the GM value of $R_{\omega}=3$. Additional finescale shear estimates were collected with a mooring during the experiment, and temporally variable shear intensity was observed, sometimes attaining values far exceeding GM [Ledwell, Watson, and Law (1998)].

The nature of the measurements may make the separation of sub-inertial band and internal-wave band signals difficult [Polzin et al. (2003)]. Note that although NATRE internal-wave strain measurements were consistent with rms $\partial_{z} c^{\prime} \sim 0.02 \mathrm{~s}^{-1}$, observed $\partial_{z} c^{\prime}$ values exceeded this by up to a factor of two in individual profiles, stemming from "static finestructure" that was removed before estimation of highfrequency strain (i.e., internal-wave strain). This means that although the ratio of rms $\partial_{z} u_{r}$ to rms wave-induced $\partial_{z} c^{\prime}$ exceeded the GM prediction, the overall ratio of $\partial_{z} u_{r}$ to $\partial_{z} c^{\prime}$ effects may, by chance, be closer to that given by GM.

\section{CONCLUSION}

It has been shown that the ray-refracting effects of vertical shear of horizontal currents in the ocean can have magnitudes exceeding one-quarter those of strain-induced sound speed perturbations. Ratios of rms shear to strain effects are up to 0.25 in the spring 1993 NATRE observations [Duda and Jacobs (1995)], and of that same magnitude for other data sets [Polzin et al. (2003)]. Because ocean sound-speed perturbations are widely believed to be large enough to in- 
fluence ray geometry in long-range propagation, the shear is thus large enough to do the same. This value of 0.25 in the upper few hundreds of meters in the ocean exceeds values of 0.13-0.17 for those depths computed earlier from an internal-wave model [Worcester (1977)], and exceeds values of $0.08-0.15$ similarly computed here. Thus, precise study of long-range acoustics may require that shear effects be included rather than neglected. Finally, the analysis shown here uses angle-depth ray geometry equations, rather than other canonical variable or vector forms, which place all of the effects into a single equation, simplifying the comparison of shear, strain, and mean-profile effects.

\section{ACKNOWLEDGMENTS}

This work was funded by grants from the U.S. Office of Naval Research. Helpful discussions with John Colosi are acknowledged. Comments made by the anonymous reviewers helped to greatly improve this work. This is WHOI contribution 11278

Beron-Vera, F. J., and M. G. Brown (2003), "Ray stability in weakly rangedependent sound channels," J. Acoust. Soc. Am. 114, 123-130.

Bowlin, J. B., J. L. Spiesberger, T. F. Duda, and L. F. Freitag (1993), Ocean acoustical ray-tracing software RAY, Woods Hole Oceanographic Institution, WHOI Tech. Report 93-10, Woods Hole, MA.

Brown, M. G., J. A. Colosi, S. Tomsovic, A. L. Virovlyansky, M. A. Wolfson, and G. M. Zaslavsky (2003), "Ray dynamics in long-range deep ocean sound propagation," J. Acoust. Soc. Am. 113, 2533-2547.

Brown, M. G., and J. Viechnicki (1998), "Stochastic ray theory for longrange sound propagation in deep ocean environment," J. Acoust. Soc. Am. 104, 2090-2104.

Colosi, J. A. (2005), "Geometric sound propagation through an inhomogeneous and moving ocean: Scattering by small scale internal wave currents," J. Acoust. Soc. Am. (to be published).

Colosi, J. A., S. M. Flatté, and C. Bracher (1994), "Internal-wave effects on $1000 \mathrm{~km}$ oceanic acoustic pulse propagation: Simulation and comparison with experiment," J. Acoust. Soc. Am. 96, 452-468.

Colosi, J. A., E. K. Scheer, S. M. Flatté, B. D. Cornuelle, M. A. Dzieciuch, W. H. Munk, P. F. Woecester, B. M. Howe, J. A. Mercer, R. C. Spindel, K. Metzger, T. G. Birdsall, and A. B. Baggeroer (1999), "Comparisons of measured and predicted acoustic fluctuations for a $3250 \mathrm{~km}$ propagation experiment in the eastern North Pacific Ocean," J. Acoust. Soc. Am. 105 3202-3218.

Duda, T. F., and C. S. Cox (1989), "Vertical wave number spectra of veloc- ity and shear at small internal wave scales," J. Geophys. Res. 94, 939950.

Duda, T. F., and D. C. Jacobs (1995), "Comparison of shear measurements and mixing predictions with a direct observation of diapycnal mixing in the Atlantic thermocline," J. Geophys. Res. 100, 13,481-13,498.

Flatté, S. M. (1983), "Wave propagation through random media: Contributions from ocean acoustics," Proc. IEEE 71, 1267-1294.

Franchi, E. R., and M. J. Jacobson (1972), "Ray propagation in a channel with depth-variable sound speed and current," J. Acoust. Soc. Am. 52, 316-331

Garrett, C. J. R., and W. H. Munk (1975), "Space-time scales of internal waves: A progress report," J. Geophys. Res. 80, 291-297.

Godin, O. A., and A. G. Voronovich (2004), "Fermat's principle for nondispersive waves in non-stationary media," Proc. R. Soc. London, Ser. A 460, 1631-1647.

Gregg, M. C. (1989), "Scaling turbulent dissipation in the thermocline," J. Geophys. Res. 94, 9686-9698.

Lamancusa, J. S., and P. A. Daroux (1993), "Ray tracing in a moving medium with two-dimensional sound speed variation and application to sound propagation over terrain discontinuities," J. Acoust. Soc. Am. 93, $1716-1726$.

Ledwell, J. R., A. J. Watson, and C. S. Law (1998), "Mixing of a tracer in the pycnocline," J. Geophys. Res. 103, 21,499-21,529.

Levitus, S. (1982), A Climatological Atlas of the World Ocean, NOAA Prof. Pap. 13, Princeton, NJ.

Munk, W., P. Worcester, and C. Wunsch (1995), Ocean Acoustic Tomography (Cambridge University Press, New York).

Nash, J. D., E. Kunze, J. M. Toole, and R. W. Schmitt (2004), "Internal tide reflection and turbulent mixing on the continental slope," J. Phys. Oceanogr. 34, 1117-1134.

Ostashev, V. E. (1997), Acoustics in Moving Inhomogeneous Media (E \& FN Spon, London)

Pinkel, R. (1984), "Doppler sonar observations of internal waves: The wavenumber-frequency spectrum," J. Phys. Oceanogr. 14, 1249-1270.

Pinkel, R. (1985), "A wavenumber-frequency spectrum of upper ocean shear," J. Phys. Oceanogr. 15, 1453-1469.

Polzin, K. L., E. Kunze, J. M. Toole, and R. W. Schmitt (2003), "The partition of finescale energy into internal waves and subinertial motions," J. Phys. Oceanogr. 33, 234-248.

Polzin, K. L., J. M. Toole, and R. W. Schmitt (1995), "Finescale parameterizations of turbulent dissipation,” J. Phys. Oceanogr. 25, 306-328.

Simmen, J., S. M. Flatté, and G.-Y. Wang (1997), "Wavefront folding, chaos, and diffraction for sound propagation through ocean internal waves," J. Acoust. Soc. Am. 102, 239-255.

Smith, K. B., M. G. Brown, and F. D. Tappert (1992), "Ray chaos in underwater acoustics," J. Acoust. Soc. Am. 91, 1939-1949.

Virovlyansky, A. L. (2003), "Ray travel times at long ranges in acoustic waveguides,” J. Acoust. Soc. Am. 113, 2523-2532.

Worcester, P. F. (1977), "Reciprocal acoustic transmission in a midocean environment," J. Acoust. Soc. Am. 62, 895-905. 